\title{
Reserve metabolism and ubiquitin proteasome system are involved in germination progress of wheat grains
}

\author{
W.D. ZHANG ${ }^{1,2}$, L. WANG ${ }^{2}$, Q.R. GAO ${ }^{1,2 *}$, Y. WANG ${ }^{1}$, and Y.Z. WANG ${ }^{1}$ \\ State Key Laboratory of Crop Science, Shandong Agricultural University, Taian City, \\ Shandong Province 271018, P.R. China ${ }^{1}$ \\ Agronomy College, Shandong Agricultural University, Taian City, Shandong Province 271018, P.R. China ${ }^{2}$
}

\begin{abstract}
To further elucidate the molecular mechanism of the germination in common wheat, a differential proteomic analysis was completed between the $\mathrm{F}_{1}$ hybrid RS5322 and its parental lines (RS53 and SN22) using isobaric tags for relative and absolute quantitation (iTRAQ) based strategy. Rapid grain germination and superior young seedling growth were observed in the $F_{1}$ hybrid line. A total of 807 differentially abundant proteins (DAPs) were identified by iTRAQ analysis of grains at $48 \mathrm{~h}$ after imbibition in distilled water. Bioinformatics analysis shows that 638 DAPs were annotated in 38 Gene Ontology functional groups, 764 DAPs were classified into 23 clusters of orthologous groups of protein categories, and 538 DAPs were enriched in 65 Kyoto encyclopedia of genes and genomes pathways. Real time quantitative PCR of 12 genes encoding different important proteins showed certain transcriptional and translational expression similarities during grain development. In the $\mathrm{F}_{1}$ hybrid, the DAPs were particularly those involved in starch and sugar metabolism, protein metabolism, protein modification, and ubiquitin proteasome system (UPS). It was speculated that UPS might be responsible for a high germination ability in the $\mathrm{F}_{1}$ hybrid by regulating storage substance metabolism. The DAPs identified in this study provide a scope for improving the grain germination trait in agricultural crops.
\end{abstract}

Additional key words: differentially abundant proteins, $\mathrm{F}_{1}$ hybrid, iTRAQ, seed imbibition.

\section{Introduction}

Heterosis is a phenomenon whereby $F_{1}$ offspring can display improved or increased function of some biological qualities that exceed those observed in their parents. The heterotic traits of an offspring are enhanced as a result of mixing the genetic contributions of its parents (Chen et al. 2015).

In plants, this phenomenon has been exploited for improvement of phenotypic and agronomic traits such as germination, growth, resistance to biotic/abiotic stresses, and yield (Fu et al. 2015). The hybrid utilization in maize, oil seed crops, and rice has greatly promoted production in the world (Premlatha et al. 2010, Mistry et al. 2016, Wang et al. 2017). The seed production cost of hybrid cultivar is generally higher than that of inbred cultivar. The seed germination quality directly affects the promotion and utilization of hybrid (Colombo et al. 2017, Derer et al. 2018). Therefore, it is very important to study the characteristic of seed quality and germination mechanism in hybrid cultivars.

Maize grains were used to study protein profile differences of seed germination between hybrid and its parents by proteomics based on two-dimensional electrophoresis. The results of $\mathrm{Fu}$ et al. (2011) indicated that dominance, partial dominance, and overdominance of the different proteins played roles in regulating seed germination in maize. The most significant are those proteins involved in germination-related hormone signal transduction, especially the abscisic acid (ABA) and gibberellin (GA) regulation networks. The results of Meena et al. (2018) indicated that the differentially

Submitted 22 August 2019, last revision 31 December 2019, accepted 10 January 2020.

Abbreviations: ABA - abscisic acid; COG - clusters of orthologous groups; DAP - differentially abundant protein; ER - endoplasmic reticulum; GA - gibberellin; GAPDH - glyceraldehyde-3-phosphate dehydrogenase; GO - gene ontology; GPAT - glucose-1-phosphate adenylyltransferase; HAI - hours after imbibition; iTRAQ - isobaric tags for relative and absolute quantitation; KEGG - Kyoto encyclopedia of genes and genomes; MS/MS - tandem mass spectrometry; NCED - 9-cis-epoxycarotenoid dioxygenase; NDHF NAD(P)H-quinoneoxidoreductase subunit 5; PSMD - 26S proteasome non-ATPase regulatory subunit; TCA cycle - tricarboxylic acid cycle; UPS - ubiquitin proteasome system.

Acknowledgements: This work was supported by grants from the Creation and Application of Strong Heterosis of Hybrid Wheat in Huanghuai Wheat Region (2016YFD0101602), which is a subject of National Key Research \& Development Program (China) "Seven Crops Breeding". The female parent RS53 were kindly provided by Dr. Liu Hongwei in the Collaboration group of this program.

* Corresponding author; e-mail: gaoqrsdau@126.com 
abundant proteins, particularly involved in metabolic and energy processes, as well as hormone biosynthesis in the $F_{1}$ hybrid, might be responsible for heterotic seed germination in this hybrid.

The germination is considered an important critical stage in the crop life cycle. Seed germination involves a series of events, which begins with water imbibition by dry seeds followed by the extrusion of the radicle towards the end. The uniform performance of crop germination and the subsequent healthy seedlings are vital factors for crop production and are of both economical and ecological importance (Lamichhane et al. 2018). The plant seed is the reservoir of saccharides, oils, and proteins, which maintain its viability during dormancy and provides primary substances for the growth of the embryo during germination (Han et al. 2014). The degradation of seed storage is a complex physiological process, and many physiological factors or pathways are involved in germination mechanisms.

So far, there are many studies on the germination characteristics of plant seeds, including global protein expression trends (Jacobsen et al. 2013, Zikova et al. 2013), specific protein function, storage materials degradation, responses to stress, and post-transcriptional modification (Novikova et al. 2014, Wei et al. 2015, Eckstein et al. 2016, Singarayer et al. 2018), and some research focused on specific organs and developmental stages at the molecular level (Long et al. 2012).

Wheat (Triticum aestivum L.) is the second most widely produced crop in the world, and its production is considered vital for global food security (Taki et al. 2018). Several wheat hybrid cultivars have been developed and grown commercially (Sun et al. 2017, Tikhenko et al. 2017); however, seldom attempts have been made to investigate germination characteristics at the translational level in wheat hybrid seeds. In this study, differentially abundant proteins (DAPs) during grain germination were identified using isobaric tags for relative and absolute quantitation (iTRAQ) approach in a high-yielding grown $\mathrm{F}_{1}$ wheat hybrid (RS5322) by comparing it with its parental lines (RS53 and SN22). We investigated the divergence of DAPs involved in physiological mechanisms in seed germination. Furthermore, the transcriptions of genes corresponding to some DAPs were also analyzed using real time quantitative PCR. This study added more information on the DAPs associated with higher seed germination ability in wheat $F_{1}$ hybrids and broadened the knowledge of molecular processes in $\mathrm{F}_{1}$ grain germination progress.

\section{Materials and methods}

Plants and treatments: Grains of wheat (Triticum aestivum L.) $\mathrm{F}_{1}$ hybrid (RS 5322) were generated by hand pollination from its female parent (RS53) and male parent Shannong 22 (SN22) and the grains were harvested at physiological maturity in June 2017 on the experimental farm of Shandong Agricultural University, Taian, China (E $117^{\circ} 09^{\prime}, \mathrm{N} 36^{\circ} 09^{\prime}$ ). The RS53 was friendly provided by Heterotic Crop Utilization Laboratory, Institute of
Crops, Chinese Academy of Agricultural Sciences, Beijing, China. The SN22 was developed and preserved in our laboratory. The mass of 1000 grains and the germination characteristics of the $F_{1}$ hybrid and its parents were represented an average of three replicates. The grains were imbibed in distilled water in the dark at $20 \pm 2{ }^{\circ} \mathrm{C}$ for $12 \mathrm{~h}$ and then they were placed with the embryo side down on moist three-layered filter paper in a Petri dish for germination under a 12-h photoperiod, an irradiance of $250 \mu \mathrm{mol} \mathrm{m} \mathrm{m}^{-2} \mathrm{~s}^{-1}$, day/night temperatures of $25 / 20{ }^{\circ} \mathrm{C}$, and a relative humidity of $75 \%$. Grains were examined at regular intervals for determining the onset of germination and were considered as germinated based on the emergence of the visible radicle. The germination rate was recorded from 12 to $96 \mathrm{~h}$ after imbibition (HAI). The germination experiments were repeated three times with each experiment consisting of five replicates and 200 grains were used in each replicate. For proteomics analysis, grains at $48 \mathrm{HAI}$ were used for analyzing the differences in the protein pattern of the $F_{1}$ hybrid in comparison with those of its parental lines.

Sample preparation and ITRAQ labeling: Protein preparation was conducted according to Ma et al. (2014) with minor modifications. Grain samples were ground into fine powder in liquid nitrogen using a mortar and pestle, and $100 \mathrm{mg}$ samples were extracted with $1 \mathrm{~cm}^{3}$ of extraction buffer [50 mM Tris-HCl $(\mathrm{pH} 8.0)$, $0.1 \mathrm{M} \mathrm{KCl}, 5 \mathrm{mM}$ EDTA, $30 \%$ (m/v) sucrose] containing $1 \mathrm{mM}$ phenylmercuricsulphonium fluoride for $3 \mathrm{~h}$. After centrifuging at $15000 \mathrm{~g}$ for $20 \mathrm{~min}$, supernatants were transferred to new tubes. Five-fold volumes of $10 \%(\mathrm{~m} / \mathrm{v})$ cold trichloroacetic acid in acetone were added to the supernatants and stored at $-20{ }^{\circ} \mathrm{C}$ for $2 \mathrm{~h}$, followed by centrifugation at $15000 \mathrm{~g}$ for $20 \mathrm{~min}$. The pellets were rinsed with $90 \%(\mathrm{v} / \mathrm{v})$ cold acetone, left at $-20{ }^{\circ} \mathrm{C}$ for $40 \mathrm{~min}$, and then centrifuged at $15000 \mathrm{~g}$ for $5 \mathrm{~min}$. This rinse step was repeated three times. After freeze drying, the final pellets were stored at $-80^{\circ} \mathrm{C}$ or analyzed instantly.

Protein samples were incorporated into $500 \mathrm{~mm}^{3}$ of STD buffer $[4 \%(\mathrm{~m} / \mathrm{v})$ sodium dodecylsulphate, $150 \mathrm{mM}$ Tris- $\mathrm{HCl}, 1 \mathrm{mM}$ dithiotreitol, $\mathrm{pH}$ 8.0], and incubated in boiling water for $8 \mathrm{~min}$, then subjected to ultra-sonication for 10 times (duration: $4 \mathrm{~min}$; time interval: $4 \mathrm{~min}$ ). After centrifugation at $15000 \mathrm{~g}$ for $60 \mathrm{~min}$, protein concentrations were determined by Bradford method. About 150 $\mu \mathrm{g}$ protein samples were diluted with $150 \mathrm{~mm}^{3}$ of UA buffer ( $8 \mathrm{M}$ urea, $150 \mathrm{mM}$ Tris- $\mathrm{HCl}, \mathrm{pH} 8.0$ ), centrifuged at $15000 \mathrm{~g}$ for $30 \mathrm{~min}$, and then $150 \mathrm{~mm}^{3}$ of UA buffer was added and centrifuged for another $30 \mathrm{~min}$. After adding $100 \mathrm{~mm}^{3}$ of UA buffer with $50 \mathrm{mM}$ iodoacetamide, the samples were incubated in darkness for $30 \mathrm{~min}$, and then centrifuged at $15000 \mathrm{~g}$ for $20 \mathrm{~min}$; this was repeated twice. Then, $100 \mathrm{~mm}^{3}$ of DS buffer $(50 \mathrm{mM}$ triethylammonium bicarbonate at $\mathrm{pH}$ 8.5) were added and centrifuged at $15000 \mathrm{~g}$ for $20 \mathrm{~min}$. This step was repeated twice and then $40 \mathrm{~mm}^{3}$ trypsin solution (2 $\mu \mathrm{g}$ trypsin from Promega in $0.04 \mathrm{~cm}^{3}$ of DS buffer) was added. The samples were incubated at $37^{\circ} \mathrm{C}$ for about $16-18 \mathrm{~h}$. The resulting peptides were collected by centrifugation and the peptide content 
was tested by Bradford method. The iTRAQ labeling of $100 \mu \mathrm{g}$ peptide samples from hybrid and its parental lines grains was performed using three iTRAQ Reagent 4-plex kits according to the manufacturer's protocol (AB Sciex, Foster City, CA, USA). Three biological replicates were iTRAQ-labeled. Protein samples of each line were equally mixed as an inner standard and labeled with 114, 115, 116, and 117 tags. Three 4 plex aApplications kits were used to label the samples in this study (Fig. 1 Suppl.).

Liquid chromatography mass spectrometry/mass spectrometry and data analysis: The iTRAQ labeled and pooled peptide samples were analyzed on a tandem mass spectrometer $Q$ Exactive equipped with an Easy $n L C$ autosampler (Thermo Fisher Scientific, San Jose, CA, USA). Survey scans were acquired at resolution of 70000 at $\mathrm{m} / \mathrm{z} 200$, and resolution for HCD spectra was set to 17500 at $\mathrm{m} / \mathrm{z}$ 200; the mass window was $2 \mathrm{Da}$ for precursor ion selection; normalized collision energy was $30 \mathrm{eV}$; and dynamic exclusion duration was $60 \mathrm{~s}$. Five $\mu \mathrm{g}$ of the peptide mixture was loaded on a packed capillary tip (C18-reversed phase column with $15 \mathrm{~cm}$ long, $75 \mu \mathrm{m}$ inner diameter) with RP-C18 $5 \mu \mathrm{m}$ resin and washed in buffer $\mathrm{A}(0.1 \%, \mathrm{~m} / \mathrm{v}$, formic acid in water $)$. After washing, the peptide mixture was separated with a gradient of buffer $\mathrm{B}(0.1 \% \mathrm{v} / \mathrm{v})$ formic acid in acetonitrile at a flow rate of $0.25 \mathrm{~mm}^{3} \mathrm{~min}^{-1}$. A data-dependent "top 10" method was used to get the most abundant precursor ions (mass range $300-1800 \mathrm{~m} / \mathrm{z}$ ). For protein identification, the MS raw files were processed by Mascot 2.4 (Matrix Science, 2012) and Proteome Discoverer 2.2 (Thermo Fisher Scientific, Shanghai, China). The acquired MS/ MS spectra were automatically searched against the uniprot_pooideae_108267.fasta, and the total number of protein sequences used in this database was 108267 . A unique protein with at least two unique peptides, with a false discovery rate $<0.01$, was qualified for further quantification data analysis. The parameters were set as: a peptide mass tolerance of $\pm 20 \mathrm{ppm}$, fragment mass tolerance of $0.1 \mathrm{Da}$, and number of allowed missed tryptic cleavage sites of 2. Protein quantification was based on the total intensity of the assigned peptides. The average of three labelled samples mixes was used as reference, based on the weighted average of the intensity of report ions in each identified peptide. When the abundance of a protein showed a difference corresponding to a $>1.6$-fold or $<0.625$-fold change in $\mathrm{F}_{1}$ compared with its parental line, and has a statistical significance $(P \leq 0.05)$, the protein was considered to be a differential abundance protein.

The functional annotations of the DAPs identified between RS5322 and its parents were performed using the Blast2GO program against the non-redundant protein database $(N R ; N C B I)$. Functional classification of proteins by clusters of orthologous groups (COG) database (http:// www.ncbi.nlm.nih.gov/COG/) was performed on all identified DAPs using the Blastx 2.2.26+ software in the STRING 9.8 database. Then, all identified DAPs were mapped to a pathway in the KEGG database using Blastx/ Blastp 2.2.26+. A $P$-value $\leq 0.05$ was used as the threshold to judge the significance of $G O, C O G$, and $K E G G$ pathway analysis results.
Extraction of RNA and real-time quantitative PCR analysis: Total RNA of wheat grains were extracted using the RN38-EASY spin Plus Plant RNA kit (Aidlab, Beijing, China) according to the manufacturer's instructions. Genomic DNA was removed by digesting each sample (20 - $50 \mu \mathrm{g}$ of total RNA) with DNase I (Promega, Madison, WI, USA). According to the manufacturer's instructions, approximately $1 \mu \mathrm{g}$ RNA was reverse transcribed to cDNA using SuperScript III (Invitrogen, USA). Primer pairs for qPCR analysis (Table 3 Suppl.) were designed by the Primer3Plus program (http://www.bioinformatics. nl/cgibin/primer3plus/primer3plus.cgi) and checked by blasting primer sequences in the NCBI database (http://www.ncbi. nlm.nih.gov/tools/primerblast/index.cgi?LINK_LOC = BlastHome), and all primers were specifically consistent with the respective sequence of its targeted gene. All qPCRs were carried out using SYBR Green PCR Master Mix (Roche Applied Science, Penzberg, Germany) on an ABI 7500 real-time PCR system (Applied Biosystems, Foster City, CA, USA) with three replicates. Data were processed using the $2^{-\Delta \Delta \mathrm{Ct}}$ method. ADP-ribosylation factor gene was used as reference for normalization.

Statistical analysis: Statistical analyses were performed with the SPSS version 17.0 software package for Windows (IBM SPSS Statistics). Data are presented as mean values $\pm \mathrm{SD}$ of independent experiments. Differences between treatments in this research were analyzed by one-way analysis of variance (ANOVA), and comparisons among means were made using the Duncan's test calculated at $P \alpha=0.05$.

\section{Results}

The germination pattern was examined in a wheat $\mathrm{F}_{1}$ hybrid (R5322) in comparison with its parental lines (Fig. 1). Germination percentage of $96.2 \%$ was achieved in the $\mathrm{F}_{1}$ hybrid at $96 \mathrm{HAI}$, which was slightly higher than those of its parents, which were 93.7 and $89.6 \%$ in the female and male parent, respectively. Germination was initiated in $\mathrm{F}_{1}$ hybrid grains at $12 \mathrm{HAI}$ with $18.5 \%$ compared with $9.2-7.8 \%$ germination rates observed in parental lines at the same time. In addition, 7-d-old seedling dry mass per plant of the $F_{1}$ hybrid $(53.6 \pm 3.7 \mathrm{mg})$ was significantly higher than those of the female parent $(46.2 \pm 5.6 \mathrm{mg})$ and the male parent $(47.6 \pm 2.8 \mathrm{mg})$. The 1000 -grain dry mass of the experimental lines were $43.3 \pm 1.9,45.3 \pm 3.1$, and $42.8 \pm 2.7 \mathrm{~g}$ in $\mathrm{F}_{1}$ hybrid, female parent, male parent, respectively. This finding indicated that the $\mathrm{F}_{1}$ hybrid with the rapid grain germination and superior seedling growth did not have the highest grain mass before germination.

In this study, iTRAQ-based quantitative proteome characterization during grain germination of the wheat hybrid RS5322 and its parental lines (RS53 and SN22) was investigated to reveal the main DAPs involved in wheat grain germination. A global profiling of quantitative proteome was obtained at $48 \mathrm{HAI}$, at which the hybrid showed significantly different germination capability compared with its parental lines. The data collected from 

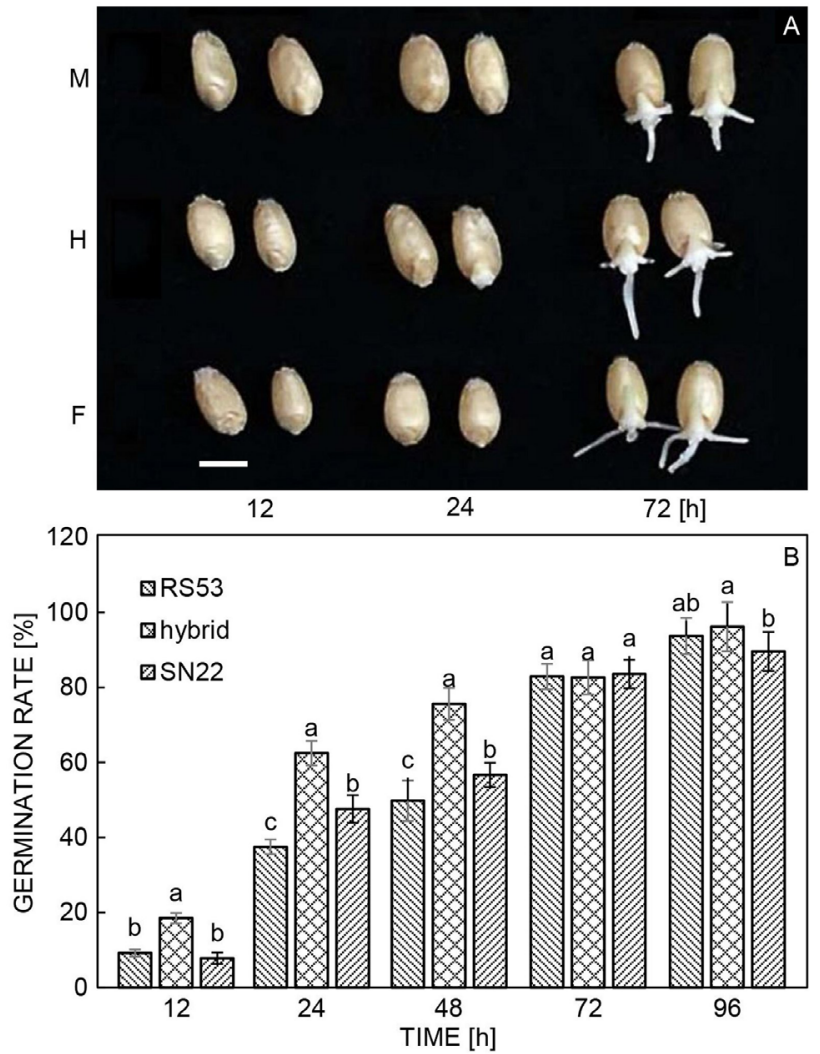

Fig. 1. The germination pattern $(A)$ and germination rate $(B)$ of grains of wheat $\mathrm{F}_{1}$ hybrid RS5322 $(\mathrm{H})$, its female parent RS53 $(\mathrm{F})$, and male parent SN22 (M) at different time points after imbibition (in distilled water for $12 \mathrm{~h}$ ). Means \pm SEs, $n=3$, different letters indicate significant differences at $\mathrm{P} \leq 0.05$ (oneway ANOVA followed by the Duncan's multiple-range tests).

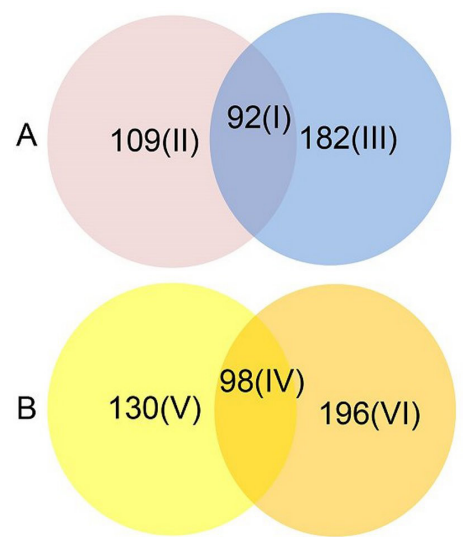

Fig. 2. Distribution of differentially abundant proteins (DAPs) between the hybrid and its parental lines. The DAPs were downregulated $(A)$ or up-regulated $(B)$ in hybrid RS 5322 compared with its female (left) or male (right) lines. Six clearly different expression patterns of the DAPs were generalized as I-VI clusters during grain germination.

these samples were analyzed using Mascot and Proteome Discovery software. Overall, 43672 spectra identified were matched to known spectra, and 12310 unique peptides and the corresponding 5651 proteins were identified. According to false discovery rate $<0.01$ and unique peptide numbers $\geq 2$, a total of 3234 non-redundant proteins were obtained.

A 1.6-fold cut-off was used to implicate significant changes in the DAPs during grain germination between RS5322 and its parental lines. Of 3234 non-redundant proteins identified, 807 showed more than 1.6-fold changes $(P \leq 0.05)$ in protein abundance between hybrid and at least one of its parental lines, therefore, these proteins were identified as DAPs. Part of DAPs in hybrid compared with its parental lines is provided in Table 4 Suppl. More DAPs and their detailed information could be found in the research laboratory's homepage (http://www.538hybridwheatsd. $\mathrm{cn} / \mathrm{B} \log / \mathrm{html} / \mathrm{blog}$ github.html) and $i \operatorname{ProX}$ (https://www. iprox.org/) with the dataset identifier IPX0001950000. The distributions of 807 DAPs and their overlapping between the hybrid and its parental lines are illustrated using the Venn diagram (Fig. 2). In general, six clearly different expression patterns during grain germination were generalized among 807 DAPs. A total of 383 DAPs were down-regulated in the hybrid compared with at least one parental line (Fig. $2 A$ ), including 92 DAPs decreased compared with both parental lines (I), while 109 and 182 DAPs, particularly down-regulated compared with the female (II) and the male line (III), respectively. As indicated in Fig. 2B, among 424 up-regulated DAPs, 98 DAPs were shared by both parental lines (IV), 130 and 196 DAPs particularly up-regulated compared with the female (V) and male line (VI), respectively.

According to the molecular functions listed on the $G O$ website, the 807 DAPs were classified into 14 functional categories (Fig. 3A). Among these functional protein categories, DAPs were involved in metabolism $(27.76 \%)$, stress/defence $(11.28 \%)$, transport $(9.91 \%)$, transcription/ translation $(8.42 \%)$, storage proteins $(7.81 \%)$, protein synthesis/assembly $(6.32 \%)$, transport $(4.99 \%)$, signal transduction $(3.84 \%)$, and unknown function $(9.29 \%)$. Subcellular localizations of the 807 identified DAPs were predicted also using $G O$ and $16.23 \%$ of DAPs were located in the plasma membranes, $14.62 \%$ in the cytoplasm, and $6.82 \%$ in the ribosomes, but $35.94 \%$ were unknown (Fig. 3B). The results of molecular functions of the 807 identified DAPs were: $21.93 \%$ of DAPs had the function of binding, $14.62 \%$ had catalytic activity, and $13.14 \%$ had structural molecule activity, but the molecular function of $16.36 \%$ DAPs were unknown (Fig. 3C).

The DAPs were further annotated based on the $C O G$ database. Of these DAPs, 766 proteins were assigned functional annotations grouped into 23 categories. A total of $13.84 \%$ of 807 proteins were involved in posttranslational modification, protein turnover, chaperones, $11.32 \%$ in general functional prediction only, 6. $81 \%$ in energy production and conversion, and $8.17 \%$ in translation (Fig. 4).

To investigate the involvement of these proteins in biological functions, $514(63.69 \%)$ DAPs were mapped to 54 pathways in the $K E G G$ database (Table 1 Suppl.). Metabolic pathways was the most represented pathway $(37.21 \%)$, followed by biosynthesis of secondary 

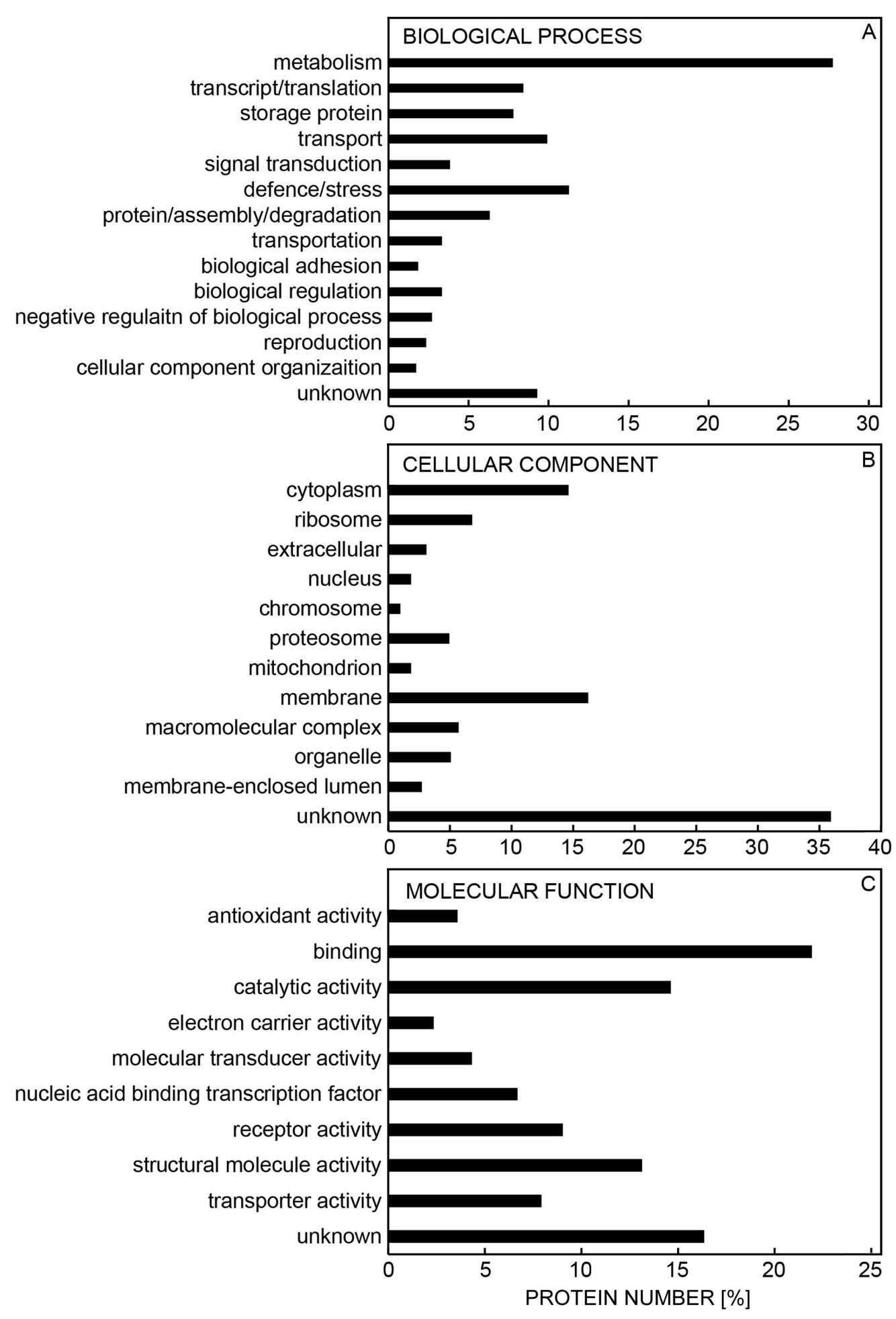

Fig. 3. Gene Ontology classification of differentially abundant proteins (DAPs) between hybrid RS5322 and its parental lines. Biological process classification $(A)$, sub-cellular localization $(B)$, and molecular function $(C)$ of the DAPs identified in the grain germination.

metabolites (18.04\%), and ribosome (5.81\%). A few DAPs were involved in pyrimidine metabolism $(0.92 \%)$ and DNA replication (0.92\%). KEGG enrichment results showed that most of these DAPs were involved in saccharide and energy metabolism pathways, such as oxidative phosphorylation, glycolysis/gluconeogenesis, and pyruvate metabolism. However, many proteins could not be annotated according to the $N C B I, G O, K E G G$, and 


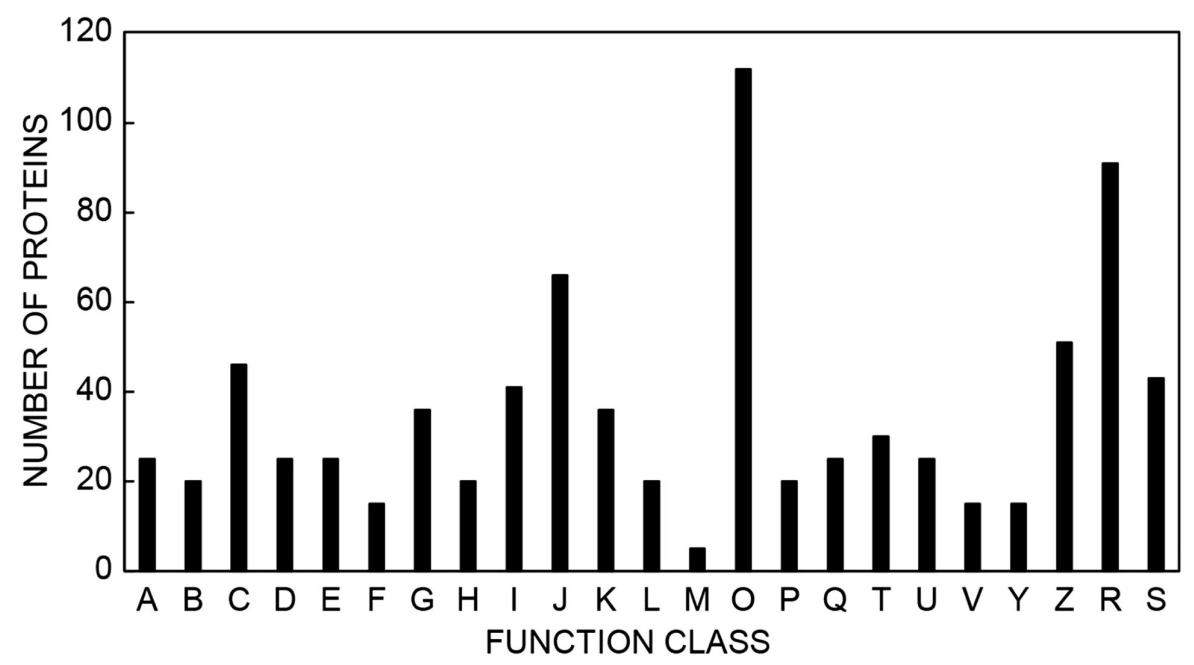

Fig. 4. Clusters of Orthologous Groups (COG) of proteins classification of differentially abundant proteins (DAPs) between hybrid RS5322 and its parental lines RS53 and SN20. Capital letters on $x$-axis represent $C O G$ categories: A - processing and modification of RNA, B - chromatin structure and dynamics, C - energy production and conversion, D - cell cycle control and mitosis, E - amino acid metabolism and transport, F - nucleotide metabolism and transport, $\mathrm{G}$ - carbohydrate metabolism and transport, $\mathrm{H}$ - coenzyme metabolism, I - lipid metabolism, J - translation, K - transcription, L - replication and repair, M - cell wall/membrane/envelope biogenesis, $\mathrm{O}$ - post-translational modification, protein turnover, chaperone functions, $\mathrm{P}$ - inorganic ion transport and metabolism, Q secondary structure, $\mathrm{T}$ - signal transduction, $\mathrm{U}$ - intracellular trafficking and secretion, $\mathrm{V}$ - defense mechanism, $\mathrm{Y}$ - nuclear structure, $\mathrm{Z}$ - cytoskeleton, $\mathrm{R}$ - general functional prediction, $\mathrm{S}$ - unknown function.

\section{$C O G$ databases.}

To verify whether the differences in protein abundance were reflected at the transcriptional level, and to confirm the authenticity and accuracy of the proteomic analysis, 12 genes (two genes selected from each of the six clusters), were analyzed by qPCR at $48 \mathrm{HAI}$ in $\mathrm{F}_{1}$ and its parental lines. From these 12 genes, 5 genes encoding $\mathrm{NAD}(\mathrm{P}) \mathrm{H}$-quinoneoxidoreductase subunit 5 (NdhF), glucose-1-phosphate adenylyltransferase (GPAT), putative PDI-like protein, 40S ribosomal protein S9, and $26 \mathrm{~S}$ proteasome non-ATPase regulatory subunit (PSMD) showed similar trends when compared with the iTRAQ analysis results from both parents. Four genes encoding $\beta$-glucosidase, triticain $\alpha, \beta$-ATP synthase subunit, and 6-fructosyltransferase, showed similar trends when compared with the iTRAQ analysis result from at least one parent. However, 3 genes encoding aspartic proteinase, thioredoxin, and protein phosphatase $2 \mathrm{~A}$ structural subunit, did not show any similar trends compared with iTRAQ analysis.

\section{Discussion}

The experiments of grain germination show that a higher germination ability was manifested in the F1 hybrid (RS5322) as evidenced by an early radicle emergence along with the vigorous growth of the radicles compared with its parental lines (RS53 and SN22, Fig. 1). The rapid germination was associated with a vigorous growth in the F1 hybrid throughout the germination period. In this study, the grain weight of the F1 hybrid was significantly lower than of the female parent but did not differ from that of the male parent. The results indicate that the rapid grain germination and superior seedling growth observed in the F1 hybrid did not correlate with grain mass in these wheat lines. The hybrid RS5322 has obvious advantages in germination rate and grain vigour, so it is suitable to be used as hybrid material in studying hybrid advantages in germination.

To further elucidate the molecular mechanism of grain germination in $\mathrm{F}_{1}$ hybrid in wheat, a differential proteomic analysis was completed between the $\mathrm{F}_{1}$ line RS5322 and its parental lines (RS53 and SN22) using iTRAQbased strategy. As a result, 807 DAPs were identified, among which, 383 were down regulated and 420 were up-regulated in $F_{1}$ compared with its parental lines (Table 4 Suppl.). Bioinformatics analysis showed that 636 DAPs were annotated in 38 functional groups, 764 DAPs were classified into $23 C O G$ categories, and 538 DAPs were enriched in $65 K E G G$ pathways. The potential roles of some DAPs in grain germination were discussed as follows.

As it is well known, the grain germination is degradation progress of storage material as it is a high energy-requiring process. Grain reserve mobilization or degradation of starch (Job 2005), protein (Maria et al. 2014, Shaik et al. 2014, Zhao et al. 2018) occurs during seed germination. The saccharide degradation is one of the basic metabolic pathways and its main physiological function is to provide energy. In this study, iTRAQ results revealed that many of the pathways contained DAPs associated with saccharide and energy metabolism (Fig. 6). As shown in Table 1 Suppl., DAPs significantly enriched in starch and sucrose metabolism (12 DAPs), glycolysis/ gluconeogenesis metabolic pathway (8 DAPs), pyruvate 

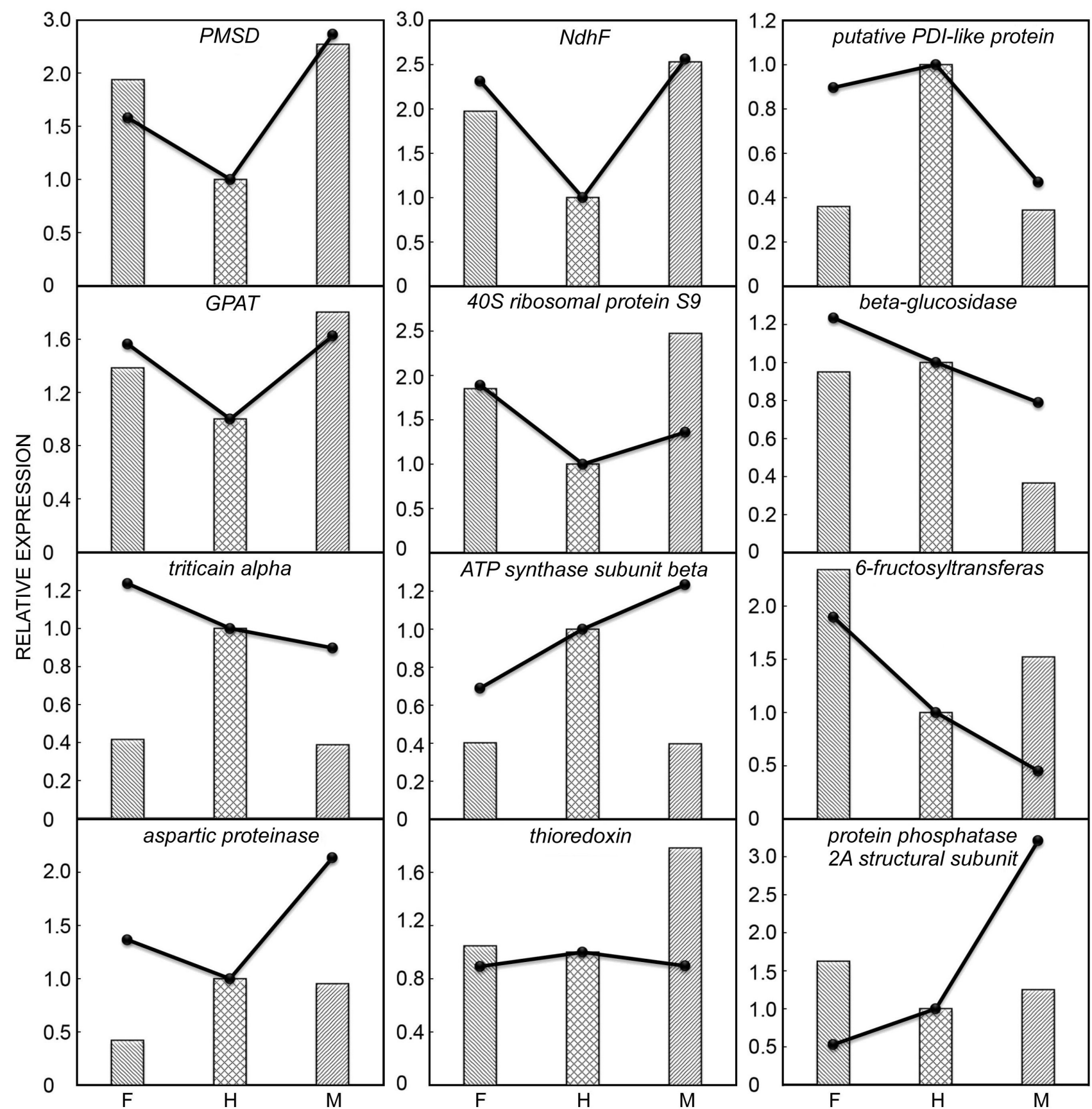

Fig. 5. Comparison of mRNA expression patterns of 12 representative differentially abundant proteins in hybrid RS5322 and its parental lines RS53 and SN22 at 48 hours after imbibition by isobaric tags for relative and absolute quantitation and by quantitative PCR. The expressions of proteins and mRNA in the hybrid were taken as controls in each protein/gene, respectively. The expression of protein and mRNA in the parents were expressed as the ratio of the parent to the hybrid. Blue columns represent protein abundance patterns, red lines represent mRNA expression patterns. F - female parent RS 53, H - hybrid line RS5322, M - male parent SN22.

metabolism (4 DAPs), TCA cycle (5 DAPs), and oxidative phosphorylation (14 DAPs). Glycolysis is the metabolic pathway that converts glucose into pyruvate. The proteome of germination between non-dormant cultivated rice and the dormant wild rice seeds was investigated and showed that the proteins involved in sucrose cleavage and glycolysis decrease, and those involved in ATP and CoQ synthesis and proteolysis increase in endosperm of germinated seeds (Xu et al. 2016). The TCA cycle is a series of chemical reactions used by all aerobic organisms to release stored energy through the oxidation of acetyl-CoA derived from sugars, fats, and proteins, into ATP and carbon dioxide. Proteomic study has been performed in dormant sunflower seeds during imbibition and germination at permissive and non-permissive temperatures for germination, respectively. The results showed that TCA cycle and glycolysis are more active in germinating seeds than in non-germinating seeds (Xia et al. 2018). Oxidative phosphorylation is the 


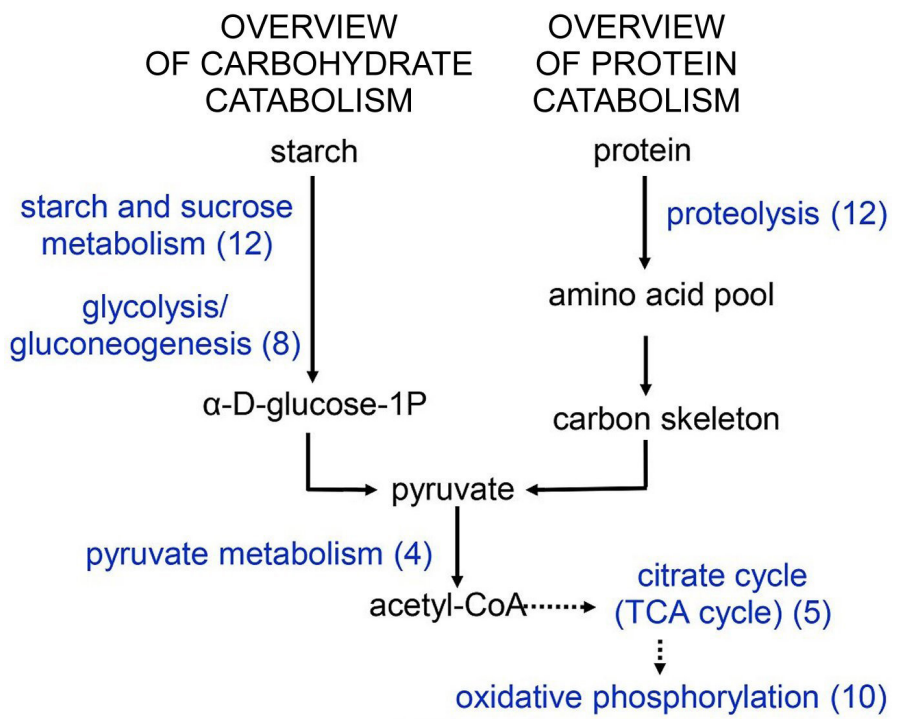

Fig. 6. Differentially abundant proteins (DAPs) involved in storage material degradation and energy metabolism in grain. The text in blue represents sugar, protein, and energy metabolism pathways; the numbers in brackets indicate the number of DAPs in the pathway.

metabolic pathway in which the mitochondria in cells utilize energy released by the oxidation of nutrients to produce ATP. Mitochondrial oxidative phosphorylation resume shortly imbibed seed after dormancy period (Pergo et al. 2011). Our results showed that many of DAPs involved in starch degradation and energy metabolism were upregulated in germinated F1 hybrid. Among these proteins, glyceraldehyde-3-phosphate dehydrogenase (GAPDH) plays an important role in glycolysis/gluconeogenesis and it is an enzyme of sugar phosphate metabolism that catalyzes the reversible interconversion of glyceraldehyde3-phosphate and 3-phosphoglycerate. GAPDH plays an important role in seed ageing and programmed cell death in P. sativum (Chen et al. 2013) and in Arabidopsis (Rajjou et al. 2008) during seed germination. Our results showed that the relative abundance of two GAPDH protein isoforms increased (2.88- and 2.31-folds) in hybrid compared with its parents, which indicated it might play a great role in wheat germination. An Arabidopsis mutant, $n d u f s 4$ (for NADH dehydrogenase [ubiquinone] fragment S subunit 4), lacking complex I of the respiratory chain, has constitutively lowered phosphorylation efficiency, delayed germination and this phenotype can be rescued by application of GA (Meyer et al. 2009). Our results showed that $\mathrm{NdhF}$, a NAD(P)H-quinone oxidoreductase subunit 5 involved in saccharide and energy metabolism pathway was significantly up-regulated in $\mathrm{F}_{1}$ hybrid compared with its parental lines. Meanwhile, a similar result was obtained by qPCR analysis (2.31- and 1.89-folds higher than those of parental lines, respectively). It can be speculated that the changed abundance of the NAD $(\mathrm{P}) \mathrm{H}$ protein might had the promotional effects during $\mathrm{F}_{1}$ hybrid germination.

Proteins play essential roles in the development of cells, organs, or tissues through the interaction with other molecules or modifications (Gutteridge and Thornton 2005), and protein synthesis and proteolysis are vital for the growth and development of plant (Murray et al. 2006). During the development of grains, storage proteins are sequestered in protein storage vacuoles, which are transformed to protein bodies at the end of maturation and drying. Published data indicate that endopeptidases, including cysteine, serine, aspartic proteases and metalloproteases, are responsible for the degradation of storage proteins during the grain development (Chrispeels et al. 1975, Cornel et al. 1994). As shown in Table 1 Suppl., 12 DAPs significantly enriched in proteolysis group and these proteins included predicted metal-dependent protease of the PAD1/JAB1 superfamily, predicted cysteine protease, subtilisin-like protease, aspartic protease, and ATP-dependent zinc metalloprotease FTSH. In previous research, four cysteine endopeptidases were detected in wheat endosperm during the initial growth after seed imbibition and the activities of all of these endopeptidases increased continuously during germination (Shi et al. 2009). Asparaginyl-endopeptidase activity was detected in endosperms of germinating wheat seeds and the high activity was found during germination (Bottari et al. 1996).

As various kinds of proteins exist in grains including globulins, gliadins, and glutenins, these endopeptidases might have different substrate specificity to the storage proteins in mature wheat endosperm. In our study, several endopeptidase involved in proteolysis were significantly up-regulated in $\mathrm{F}_{1}$ compared with its parental lines, and these important and specific endopeptidases might be tightly correlated with grain germination in $\mathrm{F}_{1}$ hybrid.

After proteolysis, amino acids are decomposed into nitrogen-containing and non-nitrogen-containing parts. The nitrogen-containing part is eventually metabolized to urea and the nitrogen-free part is $\alpha$ - ketonic acid which enters the TCA cycle. Many studies have proved that amino acid metabolisms play a great role during grain germination. Seed germination of Pinus laricio was inhibited by humic substances through affecting metabolic processes, and, in particular, the amino acid metabolism (Nardi et al. 2002). Glutamine synthetase in concert with NADH-dependent glutamate synthetase (GOGAT) 
constitute the major route of assimilation of ammonium derived from reserve mobilization and glutamic acid/ glutamine synthesis in germinating Medicago truncatula seeds (Glevarec et al. 2004). In our study, many identified DAPs were involved in pathways associated with amino acids metabolism, including cysteine and methionine metabolism (9 DAPs), glycine, serine, and threonine metabolism (7 DAPs), arginine and proline metabolism (6 DAPs) (Fig. 6). It has been reported that arginine metabolism is unbalanced in simultaneous biosynthesis and degradation of arginine, which could explain the lower accumulation of storage proteins observed during the late stages of germination (Llebres et al. 2018).

Several studies have investigated the role of ABA, GAs, and their interactions in the regulation of grain germination. ABA is the negative regulator of grain germination and promotes seed dormancy, whereas $\mathrm{GA}_{3}$ overcomes the effect of ABA by inducing germination (Gabriele et al. 2010, Liu et al. 2010, Nambara et al. 2010, Chen et al. 2017). Nine DAPs significantly enriched in hormones-related pathways were identified in our study. These pathways included DAPs in biosynthesis of plant hormones (4 DAPs) and plant hormone signal transduction (5 DAPs). NCED, 9-cis-epoxycarotenoid dioxygenase is a key enzyme in ABA biosynthesis. Previous studies have demonstrated that $L S N C E D 4$, a temperature-regulated gene, acts as a germination inhibitor (Argyris et al. 2011). In the present study, two NCED protein showed much lower expression in the $F_{1}$ hybrid compared with RS53 and SN22. Thus, lower accumulation of NCED4 in the $F_{1}$ hybrid could play an important role during germination even though the mechanism of its regulation and its precise role remain to be elucidated. On the contrary, GA3ox2-1, a 2-oxoglutarate-dependent dioxygenase, which is involved in GA biosynthesis (Pearce et al. 2015) showed much higher expression in the $\mathrm{F}_{1}$ hybrid. Moreover, AIR12, an auxin-induced in root cultures protein 12, which might be involved in the synthesis and signalling of auxins was also detected in these DAPs. The specific roles and mechanisms of these DAPs in germination needs to be further studied.

Endoplasmic reticulum ER is a subcellular organelle where proteins are folded with the help of lumenal chaperones. Correctly folded proteins are packaged into transport vesicles transporting proteins to the Golgi complex. Misfolded proteins are retained within the ER lumen in complex with molecular chaperones and they are directed toward degradation through the proteasome (Swanton et al. 2003). The ubiquitin/proteasome system (UPS) is a highly regulated mechanism of intracellular protein degradation and turnover. Through the concerted actions of a series of enzymes, proteins are marked for proteasomal degradation by being linked to the polypeptide co-factor, ubiquitin (Eleanor et al. 2018). Proteasomes are important proteases in eukaryotes and regulate many cellular processes, including metabolism, cell cycle, and proteolysis of regulatory proteins (Salomons et al. 2010), and the optimal proteasome activities are required for seed germination in bean (Inès et al. 2014) and Arabidopsis (Chiu et al. 2016). As shown in Table 2 Suppl., 26 DAPs were significantly enriched in protein processing in ER and UPS, these metabolisms included protein processing in ER (16 DAPs), proteasome (7 DAPs), and ubiquitin mediated proteolysis (3 DAPs). In a previous study, a putative E3 ubiquitin ligase ECERIFERUM9 regulates ABA biosynthesis and response during seed germination and post germination growth in Arabidopsis (Zhao et al. 2014). In our iTRAQ results, four PSMD homologs were found to be up-regulated in the hybrid compared with its parental lines. Meanwhile, qPCR results showed the higher expression abundance of a PSMD1 in hybrid (Fig. 5). The higher amount of PSMD protein might improve the degradation of misfold proteins in seed, leading to degradation of the storage and germination. In addition, selective proteolysis of proteins mediated by the ubiquitin pathway is important in the regulation of many biological events. We found that two DAPs involved in ubiquitinmediated proteolysis were up-regulated in the hybrid compared with its parental lines, which may improve the proteolysis of misfolded proteins and affect germination in hybrid. Our study showed that many proteins involved in modification, processing and degradation of proteins were significantly accumulated in $\mathrm{F}_{1}$ hybrid, which indicated that protein processing in ER and UPS might play great roles in reserve degradation or plant hormones regulation during germination.

\section{Conclusions}

Our study led to the identification ofDAPs in water-imbibed grains of a wheat $F_{1}$ hybrid compared with its parental lines. The identified DAPs were involved in various metabolic and cellular processes; the higher accumulation of proteins related with sugar and energy metabolism and proteolysis in the $\mathrm{F}_{1}$ hybrid indicates that a higher energy supply is very important for higher germination ability. Notably, the abundance of NCED4 protein, involved in biosynthetic pathway for ABA, and acting as an inhibitor in grain germination was lower in germinated grains of the $F_{1}$ hybrid. In addition, more DAPs were found to be enriched significantly in protein processing in ER and UPS. Thus, differential accumulation of these proteins and their related metabolism processes appear to be a part of the tight regulation of molecular events associated with higher grain germination in the $\mathrm{F}_{1}$ hybrid.

\section{References}

Argyris, J., Truco, J.M., Ochoa, O.: A gene encoding an abscisic acid biosynthetic enzyme (LsNCED4) collocates with the high temperature germination locusHtg6.1 in lettuce (Lactuca sp.). - Theor. appl. Genet. 122: 95-108, 2011.

Bottari, A., Capocchi, A., Galleschi, L.: Asparaginyl endopeptidase during maturation and germination of durum wheat. - Physiol. Plant. 97: 475-480, 1996.

Chen, H., Osuna, D., Colville, L., Lorenzo, O., Graeber, K., Küster, H., Leubner, M.G., Kranner, I.: Transcriptome-wide mapping of pea seed ageing reveals a pivotal role for genes related to oxidative stress and programmed cell death. - PloS ONE 8: e78471, 2013. 
Chen, X.L., Chen, X.S.,Wu, S.J., Feng, S.Q.: Recent advances in understanding plant heterosis. - Agr. Sci. 6: 1033-1038, 2015.

Chen, Z., Kong, L., Zhou, Y.: Endosperm-specific OsPYL8 and $O S P Y L 9$ act as positive regulators of the ABA signaling pathway in rice seed germination. - Funct. Plant Biol. 44: 635645, 2017.

Chiu, R.S., Pan, S., Zhao, R.: ABA-dependent ihibition of the ubiquitin proteasome system during germination at high temperature in Arabidopsis. - Plant J. 88: 749-761, 2016.

Chrispeels, M.J., Boulter, D.: Control of storage protein metabolism in the cotyledons of germinating mung beans: role of endopeptidase 12. - Plant Physiol. 55: 1031-1037, 1975.

Colombo, N., Galmarini, C.R., Havey, M.: The use of genetic, manual and chemical methods to control pollination in vegetable hybrid seed production: a review. - Plant Breed. 136: 287-299, 2017.

Cornel, F.A., Plaxton, W.C.: Characterization of asparaginyl endopeptidase activity in endosperm of developing and germinating castor oil seeds. - Physiol. Plant. 91: 599-604, 1994.

Derer, J., Tongoona, P., MacRoberts, J., Yang, H.S., Musundire, L., Dari, S.: Effect of male planting date and female plant population on hybrid maize yield and evaluation of use of hybrid-maize simulation model for grain yield estimation in hybrid maize seed production. - J. agr. Sci. 10: 333-343, 2018.

Eckstein, A., Jagieilo-Flasinska, D., Lewandowska, A., Hermanowicz, P., Appenroth, K.J., Gabrys, H.: Mobilization of storage materials during light-induced germination of tomato (Solanum lycopersicum) seeds.- Plant Physiol. Biochem. 105: 271-281, 2016.

Eleanor, H.G.A., Steven, H.S.: The ubiquitin-proteasome system as a transcriptional regulator of plant immunity. - J. exp. Bot. 69: 4529-4537, 2018.

Fu, D.H., Xiao, M.L., Hayward, A., Jiang, G.J., Zhu, L.R., Zhou, Q.H., Li,J.Q., Zhang,M.: What is crop heterosis: new insights into an old topic. - J. appl. Genet. 56: 1-13, 2015.

$\mathrm{Fu}$, Z.Y., Jin, X.I., Ding, D., Li, Y.L., Fu, Z.J., Tang, J.H.: Proteomic analysis of heterosis during maize seed germination. - Proteomics 11: 1462-1472, 2011.

Gabriele, S., Rizza, A., Martone, J., Circelli, P., Costantino, P., Vittorioso, P.: The Dof protein DAG1 mediates PIL5 activity on seed germination by negatively regulating GA biosynthetic gene AtGA3ox 1. - Plant J. 61: 312-323, 2010

Glevarec, G., Bouton, S., Jaspard, E.: Respective roles of the glutamine synthetase/glutamate synthase cycle and glutamate dehydrogenase in ammonium and amino acid metabolism during germination and post-germinative growth in the model legume Medicago truncatula. - Planta 219: 286-297, 2004.

Gutteridge, A., Thornton, J.M.: Understanding nature's catalytic toolkit. - Trends biochem. Sci. 30: 622-629, 2005.

Han, C., He, D.L., Li, M., Yang, P.F.: In-depth proteomic analysis of rice embryo reveals its important roles in seed germination. - Plant Cell Physiol. 55: 1826-1847, 2014.

Inès, K., Chaoui, A., Jaouani, K.: Role of the ubiquitin-proteasome pathway and some peptidases during seed germination and copper stress in bean cotyledons. - Plant Physiol. Biochem. 76: 77-85, 2014.

Jacobsen, J.V., Barrero, J.M., Hughes, T.: Roles for blue light, jasmonate and nitric oxide in the regulation of dormancy and germination in wheat grain (Triticum aestivum L.). - Planta 238: $121-138,2013$.

Job, C.: Patterns of protein oxidation in Arabidopsis seeds and during germination. -Plant Physiol. 138: 790-802, 2005.

Lamichhane, J.R., Debaeke, P., Steinberg, C., You, M.P., Barbetti, M.J., Aubertot, J.N.: Abiotic and biotic factors affecting crop seed germination and seedling emergence: a conceptual framework. - Plant Soil 432: 1-28, 2018.

Llebres, M.T., Pascual, M.B., Debille, S., Trontin, J.F., Harvengt, L., Avila, C., Canovas, F.M.: The role of arginine metabolic pathway during embryogenesis and germination in maritime pine (Pinus pinaster Ait.). - Tree Physiol. 38: 471-484, 2018.

Liu, Y., Ye, N., Liu, $\mathrm{R}$ : $\mathrm{H}_{2} \mathrm{O}_{2}$ mediates the regulation of ABA catabolism and GA biosynthesis in Arabidopsis seed dormancy and germination. - J. exp. Bot. 61: 2979-2990, 2010.

Long, C., Held, M., Hayward, A.: Seed development, seed germination and seedling growth in the R50 (sym16) pea mutant are not directly linked to altered cytokinin homeostasis. - Physiol. Plant. 145: 341-359, 2012.

Lopez-Pedrouso, M., Alonso, J., Zapata, C.: Evidence for phosphorylation of the major seed storage protein of the common bean and its phosphorylation-dependent degradation during germination. - Plant mol. Biol. 84: 415-428, 2014.

Ma, C.Y., Zhou, J.W., Chen, G.X., Bian, Y.W., L., D.W., Li, X.H., Wang, Z.M., Yan, Y.M.: iTRAQ-based quantitative proteome and phosphoprotein characterization reveals the central metabolism changes involved in wheat grain development. BMC Genomics 15: 1029, 2014

Meena, R.K., Pullaiahgari, D., Gudipalli, P.: Proteomic analysis of heterotic seed germination in maize using $F_{1}$ hybrid DHM 117 and its parental inbreds. - Turkish J. Biol. 42: 345-363, 2018.

Meyer, E.H., Tomaz, T., Carroll, A.J., Estavillo, G., Delannoy, E., Tanz, S.K., Small, I.D., Pogson, B.J., Millar, A.H.: Remodeled respiration in $n d u f_{s} 4$ with low phosphorylation efficiency suppresses Arabidopsis germination and growth and alters control of metabolism at night. - Plant Physiol. 151: 2187-2187, 2009.

Mistry, P.M., Prajapati, M.B., Belhekar, P.S.: Heterosis for grain yield and yield attributing traits in rice (Oryza sativa L.). - J. agr. Res. Technol. 41: 205-213, 2016.

Murray, R.F., Harper, H.W., Granner, D.K., Mayes, P.A., Rodwell, V.W.: Harper's Illustrated Biochemistry. - Lange Medical Books/McGraw-Hill., New York 2006.

Nambara, E., Okamoto, M., Tatematsu, K., Yano, R., Seo, M., Kamiya, Y.: Abscisic acid and the control of seed dormancy and germination. - Seed Sci. Res. 20: 55-67, 2010.

Nardi, S., Muscolo, A., Sidari, M., Panuccio, M.R., Sessi, E.: Alteration of amino acid metabolism by humic substances during germination of Pinus laricio seeds. - Seed Sci. Technol. 30: 205-210, 2002.

Novikova, G.V., Tournaire-Roux, C., Sinkevich, I.A.: Vacuolar biogenesis and aquaporin expression at early germination of broad bean seeds. - Plant Physiol. Biochem. 82: 123-132, 2014

Pearce, S., Huttly, A.K., Prosser, I.M.: Heterologous expression and transcript analysis of gibberellin biosynthetic genes of grasses reveals novel functionality in the GA3ox family. BMC Plant Biol. 15: 130, 2015.

Pergo, E.M., Ishii-Iwamoto, E.L.: Changes in energy metabolism and antioxidant defense systems during seed germination of the weed species ipomoea triloba 1 . and the responses to allelochemicals. - J. chem. Ecol. 37: 500-513, 2011.

Premlatha, M., Kalamani, A.: Heterosis and combining ability studies for grain yield and growth characters in maize (Zea mays L.). - Indian J. agr. Res. 44: 62-65, 2010.

Rajjou, L., Lovigny, Y., Groot, S.P.C., Belghazi, M., Job, C.: Proteome wide characterization of seeds ageing in Arabidopsis: A comparison between artificial and natural ageing protocols. - Plant Physiol. 148: 620-641, 2008.

Salomons, F.A., Klara, A., Dantuma, N.P.: Illuminating the ubiquitin/proteasome system. - Exp. cell. Res. 316: 1289- 
$1295,2010$.

Shaik, S.S., Carciofi, M., Martens, H.J.: Starch bioengineering affects cereal grain germination and seedling establishment. J. exp. Bot. 65: 2257-2270, 2014.

Shi, C., Xu, L.L.: Characters of cysteine endopeptidases in wheat endosperm during seed germination and subsequent seedling growth. - J. Integrative Plant Biol. 51: 52-57, 2009.

Singarayer, F., Sandra, W., Alannah, K., Arunthathy, F., Kim, D., Martin, W., Bhagirath, S.C.: Seed germination response of a noxious agricultural weed Echium plantagineum to temperature, light, $\mathrm{pH}$, drought stress, salinity, heat and smoke.- Crop Pasture Sci. 69: 326-333, 2018.

Sun, C., Ruan, R.W., Yan, L.L., Hu, D., Liu, X.B., She, H.Z., Xu, M.M., Li, Z.G., Yi, Z.L., Yang, Y.H.: Effects of different row ratios of male and female parents and application amount of nitrogen fertilizer on hybrid wheat seed production and its component factors. - Asian agr. Res. 9: 69-73, 2017.

Swanton, E., Bulleid, N.J.: Protein folding and translocation across the endoplasmic reticulum membrane. - Membrane Biol. 20: 99-104, 2003.

Taki, M., Soheili-Fard, F., Rohani, A., Chen, G.N., Yildizhan, H.: Life cycle assessment to compare the environmental impacts of different wheat production systems. - J. cleaner Product. 197: 195-207, 2018.

Tikhenko, N.D., Tsvetkova, N.V., Lyholay, A.N., Voylokov, A.: Identification of complementary genes of hybrid lethality in crosses of bread wheat and rye. - Russ. J. Genet. appl. Res. 7: 153-158, 2017.

Vizcaino, J.A., Cote, R. G., Csordas, A.J., Dianes, A. Fabregat, A., Foster, J.M.: The proteomics. Identifications (PRIDE) database and associated tools: status in 2013. - Nucl. Acids
Res. 41: D1063-D1069, 2013.

Wang, L.F., Zhang, F., Fu, T.D., Shen, J.X.: A review: status and prospect of heterosis prediction in rapeseed (Brassica napus L.). - Chin. J. Oil Crop Sci. 3: 187-194, 2017.

Wei, T., He, Z.L., Tan, X.Y., Liu, X., Yuan, X., Luo, Y.F., Hu, S.N.: An integrated RNA-Seq and network study reveals a complex regulation process of rice embryo during seed germination.Biochem. biophys. Res. Commun. 464: 176-181, 2015.

Xia, Q., Maharajah, P., Cueff, G., Rajjou,L., Prodhomme, D., Gibon, Y., Bailly, C., Corbineau,F., Meimoun, P., El-MaaroufBouteau, H.: Integrating proteomics and enzymatic profiling to decipher seed metabolism affected by temperature in seed dormancy and germination. - Plant Sci. 269: 118-125, 2018.

Xu, H.H., Liu, S.J., Song, S.H.: Proteomics analysis reveals distinct involvement of embryo and endosperm proteins during seed germination in dormant and non-dormant rice seeds. - Plant Physiol. Biochem. 103: 219-242, 2016.

Zhao, H.Y., Zhang, H.M., Cui, P., Ding, F., Wang, G.C., Li, R.J., Matthew, A.J., Lü, S.Y., Xiong, L.M.: The putative E3 ubiquitin ligase ECERIFERUM9 regulates abscisic acid biosynthesis and response during seed germination and postgermination growth in Arabidopsis. - Plant Physiol. 165: 1255-1268, 2014.

Zhao, M., Zhang, H., Yan, H., Qiu, L., Baskin, C.C.: Mobilization and role of starch, protein, and fat reserves during seed germination of six wild grassland species. - Front. Plant Sci. 9: 1-11, 2018

Zikova, A., Vohradsky, J., Strakova, E., Bobek, J.: Global features of gene expression on the proteome and transcriptome levels in S. coelicolor during germination. - PLoS ONE 8: e72842, 2013. 